\title{
Factors influencing low intension detection rate in a non- invasive EEG-based brain computer interface system
}

\author{
Clifford Maswanganyi ${ }^{1}$, Chungling $\mathrm{Tu}^{2}$, Pius Owolawi ${ }^{3}$, Shengzhi Du ${ }^{4}$ \\ ${ }^{1,2,3}$ Department of Computer Systems Engineering, Tshwane University of Technology, South Africa \\ ${ }^{4}$ Department of Electrical Engineering, Tshwane University of Technology, South Africa
}

\begin{abstract}
Article Info
\section{Article history:}

Received Feb 23, 2020

Revised Mar 25, 2020

Accepted Apr 14, 2020

\section{Keywords:}

Brain computer interface Electroencephalogram

Hybrid tasks

Motor imagery

Steady State visual evoked potential

ABSTRACT

Motor imagery (MI) responses extracted from the brain in the form of EEG signals have been widely utilized for intention detection in brain computer interface (BCI) systems. However, due to the non-linearity and the nonstationarity of EEG signals, BCI systems suffer from low MI prediction rate with both known and unknown influncing factors. This paper investigates the impact of visual stimulus, feature dimensions and artifacts on MI task detection rate, towards improving MI prediction rate. Three EEG datasets were utilized to facilitate the investigation. Three filters (band-pass, notch and common average reference) and the independent component analysis (ICA) were applied on each datasets, to eliminate the impact of artifact. Three sets of features where extracted from artifact free ICA components, from which more relevant features were selected. Moreover, the selected feature subsets were incorporated into three classifiers, NB, Regression Tree and K-NN to predict four MI and hybrid tasks. K-NN classifier outperformed the other two classifies in each dataset. The highest classification accuracy is obtained in hybrid task EEG dataset. Moreover, accurately predicted EEG classes were applied to a robotic arm control.
\end{abstract}

Copyright @ 2020 Institute of Advanced Engineering and Science. All rights reserved.

Corresponding Author:

Chunling Tu,

Departement of Computer Systems Engineering,

Tshwane University of Technology,

Staatsartillerie Rd, Pretoria West, Pretoria, 0183, South Africa.

Email: duc@tut.ac.za

\section{INTRODUCTION}

Brain computer interface (BCI) systems in recent years have emerged as a breakthrough technology, capable of aiding individuals suffering from severe motor disabilities interact with the surrounding environment using only imagined movements. Brain computer interface is a process of extracting neural activities of the brain in the form of EEG signals, and transforming them into control commands utilized to control electronic equipment. However, during acquisition of EEG signals from the brain several interferences exist (such as physiological and non-physiological artifacts) which may have significant impact on the quality of recorded EEG signals. Moreover, during translation of EEG signals into control commands the dimension of extracted feature set may also have significant impact on the performance signal classifiers, mainly as a result of redundant features contained within the extracted feature set. As such low MI detection and prediction is still one of the most significant challenges in EEG based BCI systems. Recently countless techniques have been developed to address the challenge of low MI detection and prediction. [1] proposed a BCI system that utilized EEG dataset acquired from BCI competition III-a database, whereby four band-pass filters were firstly applied on EEG dataset to eliminate artifacts. Moreover, common spatial pattern technique was utilized to extract features from pre-processed EEG dataset. As such SVM was utilized to predict two MI classes from extracted features, and a highest classification accuracy of 85.5\% was achieved. [2] proposed a BCI system that utilizes EEG dataset acquired from BCI competition II database from which a $0.5 \mathrm{~Hz}-30 \mathrm{~Hz}$ 
elliptic band-pass filter was applied to eliminate artifacts. Furthermore, statistical features were extracted from pre-processed EEG dataset, whereby SVM and multi-perceptron classifiers were both applied on extracted features to predict two MI classes. The highest classification accuracy of $85 \%$ and $85.71 \%$ were obtained from both signal classifiers respectively. Multiple filters were reported by combining with one-toone classification [3]. This paper investigates the impact of visual stimulus, artifacts and feature dimension on prediction rate. To evaluate the above mentioned factors a hybrid EEG based BCI system consisting of five stages is proposed. In this case a signal acquisition stage utilized to record EEG datasets, a preprocessing stage utilized to filter and decompose EEG signals to remove noise and artifactual components, whereby artifact free ICA components are utilized to extract three sets of features from each of the three datasets during feature extraction stage. Moreover, relevant signal feature subsets with high predictive power are selected, and used as inputs to signal classifiers to predict four MI task during feature selection and classification stage respectively.

\section{RESEARCH METHOD}

\subsection{EEG signal acquisition}

A publicly available database (BCI competition IV-a) and two online recorded EEG datasets using a gtec EEG recording system were utilized [4]. BCI competition IV-a dataset was acquired from nine healthy subjects using twenty-two $\mathrm{Ag} / \mathrm{AgCl}$ channels position on the scalp according to a 10-20 electrode positioning system as illustrated in Figure 1(b), whereby EEG signals were recorded at a sampling rate of 250Hz [5]. At the beginning of the experiment a fixed cross and a two second beeping sound were utilized to notify the subjects to begin imagining, and each subject was required to performed four motor imagery task (left hand, right hand, tongue and both feet) [6]. Furthermore, two EEG datasets were non-invasively recorded from one subject at a sampling rate of $250 \mathrm{~Hz}$ using 16 electrodes. In this case imagine only EEG dataset recorded while the subject performed four motor imagery tasks as illustrated in Figure 1(a). Secondly a hybrid tasks EEG dataset recorded while the subject performed a combination of SSVEP and MI tasks. As such visual stimulus in the form of four flashing balls were displayed on an LCD monitor, while the subject performed four MI tasks [7]. For both online recorded EEG datasets at the beginning of a trial the subject was requested to imagine the required MI task for 300 seconds [8]. Moreover, a beeping sound was utilized to notify the subject to begin imagining any of the four MI tasks (left hand, right hand, tongue and both feet) [9]. As such the same process was repeated for the other three MI classes and as a result a trial lasted for twenty minutes. For hybrid EEG dataset the same experimentation paradigm as MI tasks was repeated, in this case the visual stimulus were tagged with four different frequencies $(29 \mathrm{~Hz}, 13.3 \mathrm{~Hz}, 17 \mathrm{~Hz}, 21 \mathrm{~Hz})$ corresponding to each MI task [10].

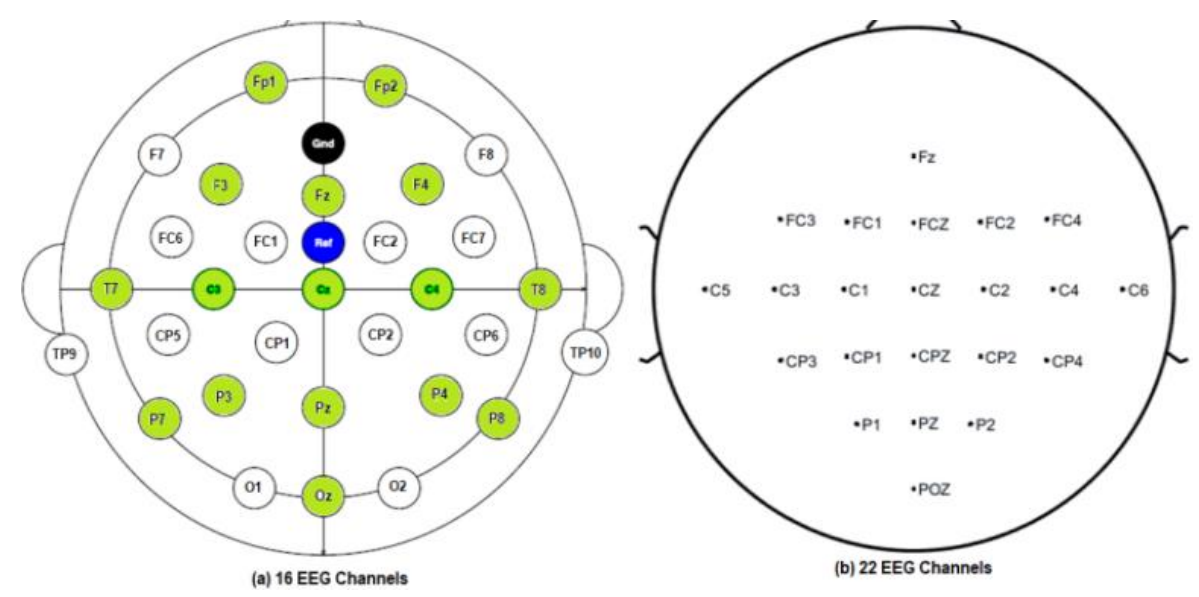

Figure 1. 10-20 electrode positioning system for online recorded EEG datasets (b) 10-20 electrode positioning system for BCI competition IV-a dataset [6]

\subsection{EEG signal preprocessing}

In this section a $0.5-60 \mathrm{~Hz}$ band-pass, $48-52 \mathrm{~Hz}$ notch and a CAR filter were firstly applied on both sets of recorded EEG data to eliminate the impact of non-physiological artifacts [11]. Subsequently, a 0.5$100 \mathrm{~Hz}$ band-pass and a $50 \mathrm{~Hz}$ notch filter was also applied on BCI competition IV-a dataset. EEGLAB was utilized to implement runICA algorithm, consequently sixteen and twenty-two ICA components were 
generated from both filtered online recorded and BCI competition EEG dataset respectively, from which artifactual components can be rejected [12]. Furthermore, sixteen ICA components acquired from imagined only dataset were manually evaluated to eliminate artifactual windows using a sliding window technique. Moreover, each component was divided into twenty blocks containing twenty windows each, then a sliding window represented by two red vertical lines was applied on each block to remove artifactual windows as illustrated in Figure. 2.
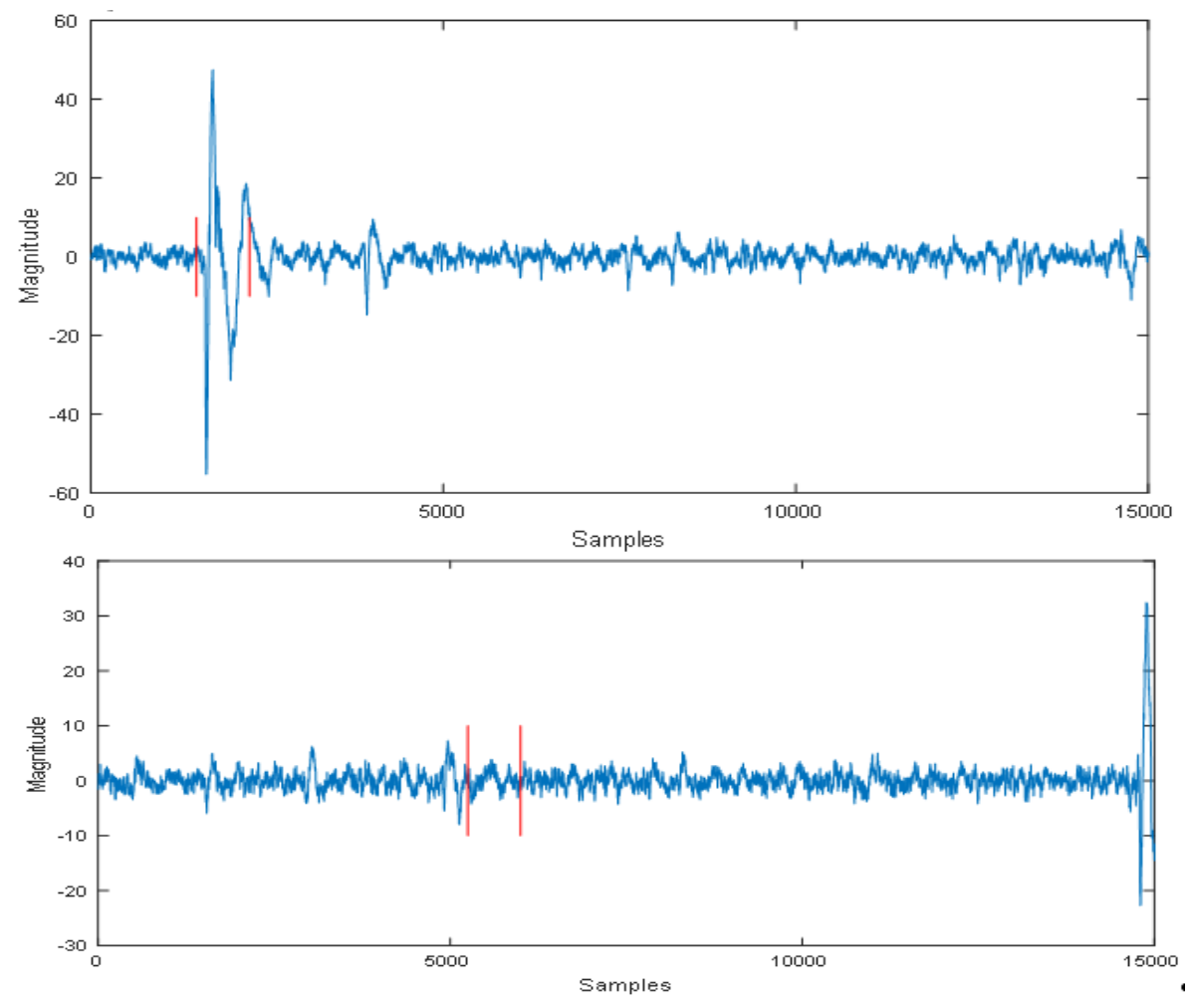

Figure 2. Sliding window approach applied on imagine only ICA components

\subsection{Feature extraction}

Three sets of features were extracted from each ICA component and component eight online recorded and BCI competition IV-a datasets respectively. Fast fourier transform (FFT) denoted by (1) was utilized to extract band-power features in the form of five frequency bands (namely delta $(\delta): 0.5-3 \mathrm{~Hz}$, theta $(\theta): 4-8 \mathrm{~Hz}$, alpha $(\alpha): 8-12 \mathrm{~Hz}$, beta $(\beta): 12-30 \mathrm{~Hz}$ and gemma $(\gamma): 30-60 \mathrm{~Hz})$ from ICA components [13-15].

$$
X(f)=F\{x(t)\}=\int x(t) e^{-2 \pi f t d t}
$$

FFT is denoted by $X(f)$ and EEG signals in time domain denoted by $x(t)$ [16]. MATLAB statistical toolbox was utilized to extract ten signal features from each of the three EEG datasets (namely mean, median, standard deviation, mean absolute deviation, skewness, kurtosis, spectral entropy and dominant frequency features (maximum frequency, maximum value, maximum ratio)) [17].

$$
\sigma=\sqrt{\frac{1}{N} \sum_{i=1}^{N}\left(x_{i}-\bar{x}\right)^{2}}
$$

In this case standard deviation $(\sigma)$ is denoted by (2), and signals within a specific window denoted by $x_{i}$. Moreover, the arithmetic mean of signals within a specific window is denoted by $\bar{x}$, with $N$ representing the data points in total within a specific window [18].

$$
M=\frac{1}{n} \sum_{i=1}^{n} x_{i}
$$

\footnotetext{
Factors influencing low intension detection rate in a non-invasive EEG-based ... (Clifford Maswanganyi)
} 
Mean of a signal $(M)$ denoted by (3), whereby signals within a specific window are denoted by $x_{i}$ and with $n$ signifying the overall samples within a window [18].

$$
\begin{aligned}
& \text { Med }_{\text {even }}=\frac{\frac{n}{2}+\left(\frac{n}{2}+1\right)}{2} \\
& \operatorname{Med}_{\text {odd }}=\frac{(n+1)}{2}
\end{aligned}
$$

Median of a signal is computed using either (4) or (5) depending on whether $n$ is even or odd, whereby the overall sample points within a certain window is denoted by $n$ [18].

$$
\begin{aligned}
& P\left(\omega_{i}\right)=\frac{1}{N}\left|X\left(\omega_{i}\right)\right|^{2} \\
& P_{i}=\frac{P\left(\omega_{i}\right)}{\sum P\left(\omega_{i}\right)} \\
& P S E=-\sum_{i=1}^{n} P_{i} \ln P_{i}
\end{aligned}
$$

Power spectral entropy (PSE) in (8) is dependent on power spectral density $P\left(\omega_{i}\right)$, and the normalized power spectral density $P_{i}$ defined both (6) and (7) respectively. Whereby $X\left(\omega_{i}\right)$ and $N$ in (6) signifies the value of signals within a certain window, and the overall samples with a certain window respectively. Moreover, the combination of all signal values acquired through the PSD is denoted by $\sum P\left(\omega_{i}\right)$ in (7) [18].

$$
M A D=\frac{1}{N} \sum_{i=1}^{N}\left|x_{n}-\bar{x}\right|
$$

As shown in (9) defines the mean absolute deviation (MAD) of a signal, whereby $x_{n}$ represents signals within a specific window. $\bar{x}$ represents the arithmetic mean of signals within a specific window and $N$ representing the data points in total within a specific window [19]. Skewness and kurtosis is defined using both (10) and (11), whereby $M$ represents the overall samples within a signal and $y$ representing subbands[20]. Wavelet packet transform (WPT) was utilized to extract 255 wavalet features from pre-processed EEG signals [21, 22].

$$
\begin{aligned}
& \emptyset=\sqrt{\frac{1}{M} \sum_{j=1}^{M} \frac{\left(y_{j}-\mu\right)^{3}}{\sigma^{3}}} \\
& \emptyset=\sqrt{\frac{1}{M} \sum_{j=1}^{M} \frac{\left(y_{j}-\mu\right)^{4}}{\sigma^{4}}} \\
& A_{i}(t)=\sum_{k=-\infty}^{\infty} A_{i-1}(k) \emptyset_{i}(t-k) \\
& D_{i}(t)=\sum_{k=-\infty}^{\infty} A_{i-1}(k) \psi_{i}(t-k)
\end{aligned}
$$

Two filters denoted by $x(t)$ were utilized to decompose signals into multiple separate coeffients through a wavelet transform, whereby $n$ represents the number of samples. A lowpass and highpas filter in the form of a wavelet and a scaling function decomposed signals into detail (13) and approximation coeffients (12) respectively [23, 24]. As such decomposed approximation coefficients in each level resulted in WPT tree comprising of both detail and approximation coefficients as illustrated in Figure 3 [25, 26].

$$
x(t)=\sum_{k=-\infty}^{\infty} A_{i-1}(k) \emptyset_{i}(t-k)+\sum_{i=1}^{L} \sum_{k=-\infty}^{\infty} A_{i-1}(k) \psi_{i}(t-k)
$$

As shown in (14) was utilized to reconstruct decomposed wavelet coefficients from which wavelet features are extracted using overlapping window approach and separated into several windows with each window size set to 750 , and each window incremented by 750 or 750 spaces apart $[27,28]$. 


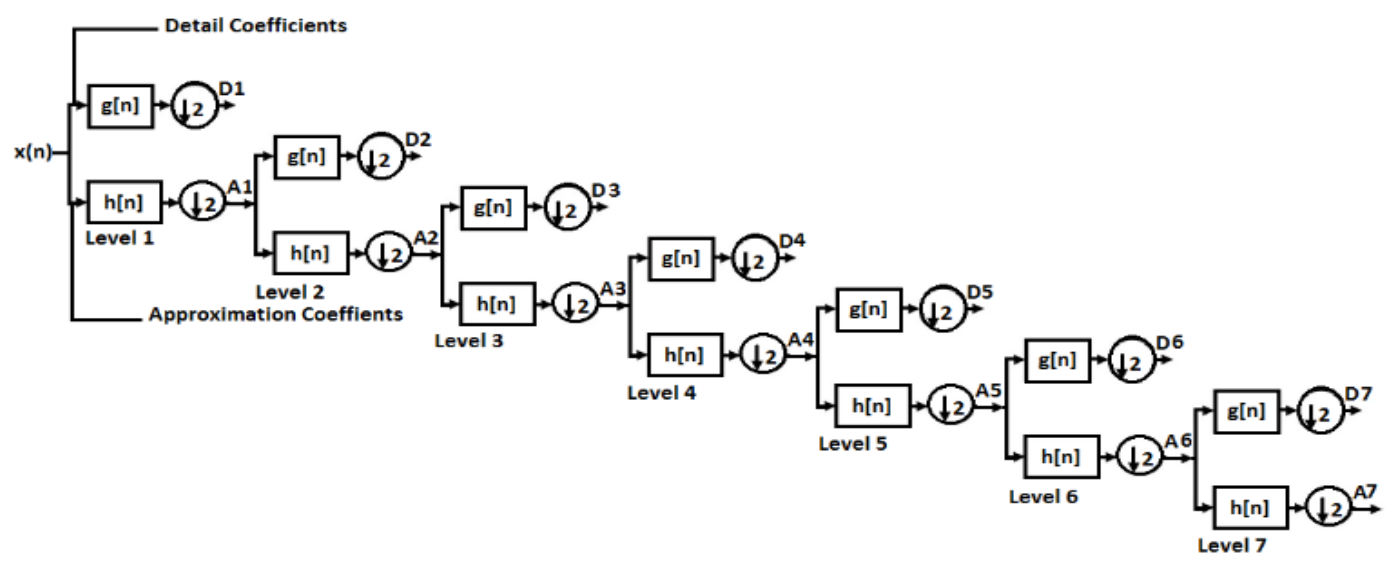

Figure 3. Seven decomposition levels using 4th order daebuchies wavelet

\subsection{Feature selection and classification}

In this section differential evolution based channel and feature selection (DEFS) algorithm was applied on extracted features, to eliminate redundant features and the challenge dimensionality [26]. In this case DEFS algorithm was utilized to train three signal classifiers and then select the best feature subsets using six input parameters. Features were divided into training and testing set, with the last column of the features matrices being the class labels. As such the speed with which features converge between the $1^{\text {st }}$ and $500^{\text {th }}$ generation was evaluated and utilized as a criterion to determine the DNF (in this case $30,50,80$ ) and PSIZE (150) parameter in which the DEFS algorithm performs at its optimal best using the error rate. The desired number of features (DNF) was set to 80 and 130 for online recorded and BCI competition IV-a dataset respectively, while the population size (PSIZE) was set to 150. Consequently, the number of iterations was set to start at zero while the number of generations (GEN) was set to 1000 as our terminating condition. Three signal classifiers (K-nearest neighbor, Naïve bayes and Regression tree) were applied on extracted features to predict both MI and hybrid tasks [29, 30]. As such the performance of each of the three signal classifiers was firstly evaluated to determine the best performing classifier, whereby the best performing classifier was then utilized during robotic arm control. Extracted feature sets were divided into test and training set incorporated into each of the three classifiers. Moreover, the best 80 and 130 feature subsets were also incorporated into each of the three signal classifiers utilized to predict both MI and hybrid tasks.

\section{RESULTS AND ANALYSIS}

\subsection{EEG data source and pre-processing}

Three sets of EEG data are utilized in the proposed BCI system. Firstly a publicly available database (BCI completion IV-a) consisting of EEG signals acquired from 22 EEG channels, subject two dataset was utilized for this experiment and the dataset consisted of a signal length of 677169 frames per epoch [8]. Secondly two online recorded datasets acquired from 16 EEG channels attached to gGEMMA sensor cap. Both datasets were recorded for 20 minutes and resulted in a signal length of 300000 frames per epoch [9]. Twenty-two ICA components acquired from BCI competition IV-a were visually inspected, whereby EEG spectrum or power spectrum of each component was evaluated [9]. As such spectral peaks at certain frequencies and dipole-like scalp maps were evaluated mainly to reject artifactual components as shown in Figure 4 and Figure 5 respectively. In this case ICA component eight was selected for further signal processing.
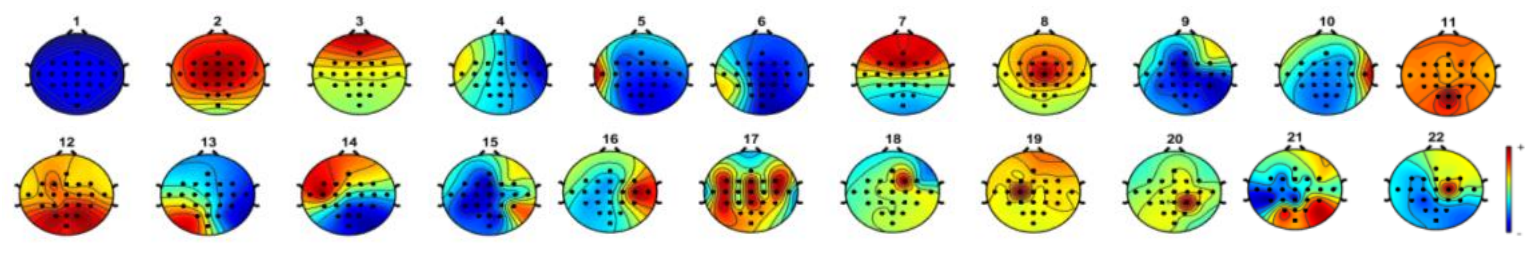

Figure 4. Twenty-two ICA components generated from BCI competition IV-a dataset

Factors influencing low intension detection rate in a non-invasive EEG-based ... (Clifford Maswanganyi) 

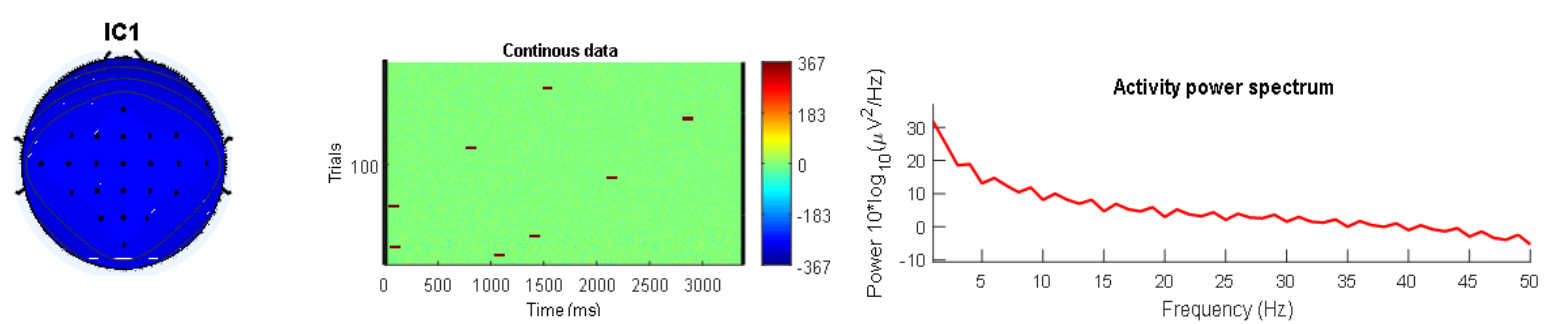

Figure 5. Component spectra and maps

A sliding window approach utilized to remove artifactual windows from all sixteen ICA components acquired from imagine only EEG dataset, whereby 2295 artifactual windows were removed from all components. As such a minimum of 53 and a maximum of 243 windows were removed per component.

\subsection{Extracted and DEFS selected feature sets}

Three sets of features were extracted from each of the three datasets namely wavelet, band-power and statistical features as illustrated in Table 1. Consequently, BCI competition IV-a and both online recorded datasets resulted in a 4590x271 and 480x271 feature matrix respectively [6]. Both 4590 and 480 represented the number of samples while 271 represented the total number of feature with the last column signifying the class labels.

Features convergence and DEFS algorithm performance is at its optimal best when the DNF is set to 80 and PSIZE to 150 as illustrated in Figure 6 [26]. As such 80 and 130 feature subsets were selected from bothonline recorded datasets and BCI competition IV-a dataset respectively as illustrated in Table 2 and Table 3 [3].

Table 1. Extracted feature sets

\begin{tabular}{|c|c|c|c|}
\hline Features & Types of features & Feature extraction algorithm & Number of features \\
\hline Band power & $\begin{array}{l}\text { delta: } 0.5-3 \mathrm{~Hz} \text {, theta: } 4-8 \mathrm{~Hz} \text {, alpha: } 8-12 \mathrm{~Hz} \text {, } \\
\text { beta: } 12-30 \mathrm{~Hz} \text {, gemma: } 30-60 \mathrm{~Hz}\end{array}$ & FFT & 5 \\
\hline $\begin{array}{l}\text { Statistical } \\
\text { Features }\end{array}$ & $\begin{array}{l}\text { Mean, Median, standard deviation, mean absolute } \\
\text { deviation, skewness, kurtosis, spectral entropy, } \\
\text { dominant frequency features (maximum frequency, } \\
\text { maximum value and maximum ratio)) }\end{array}$ & $\begin{array}{l}\text { Statistical Computation } \\
\text { (MATLAB statistical toolbox) }\end{array}$ & 10 \\
\hline Wavelet features & Wavelet coefficients & WPT & 255 \\
\hline
\end{tabular}

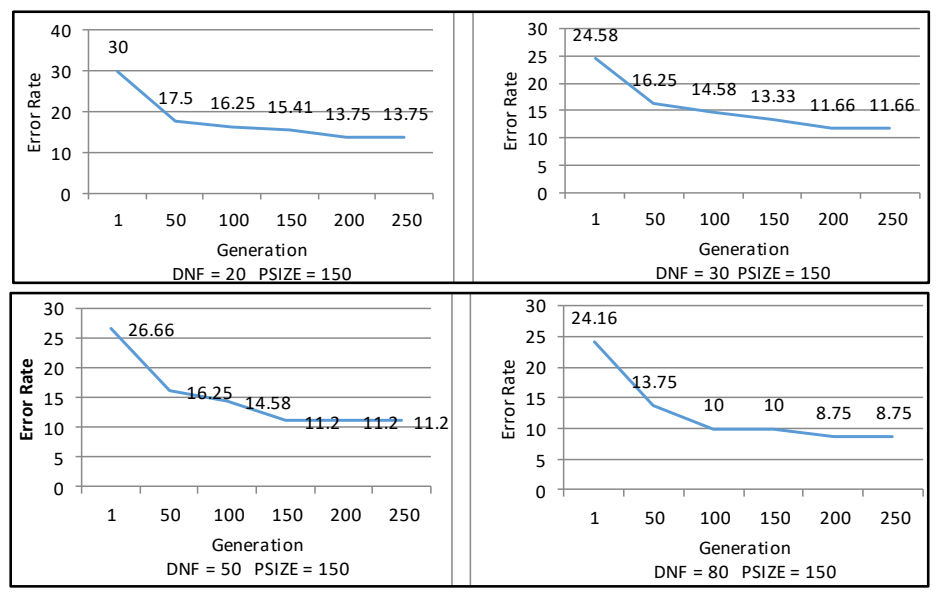

Figure 6. Feature convergence during feature selection

Table 2. 130 selected feature subsets

\begin{tabular}{ccl}
\hline Desired number of features & Length of features & Category of features(number) \\
\hline \multirow{2}{*}{130} & 4590 & $\begin{array}{l}\text { Wavelet features (123), Band-power (Gamma) (1), Statistical (6), (Mean, } \\
\text { Median, skewness, kurtosis, dominant frequency standard deviation, Maxratio) }\end{array}$ \\
\hline
\end{tabular}


Table 3. 80 selected feature subsets

Desired number of features $\quad$ Length of features $\quad$ Category of features(number)

\subsection{Feature classification}

In this section the training set, test set and the best selected feature subsets were incorporated into each of the three signal classifiers for performance evaluation (K-NN, NB and Regression tree) [25]. Consequently, K-NN classifier outperformed all classifiers obtaining a highest accuracy of $88.2 \%, 69.7 \%$ and $100 \%$ BCI competition IV-a, imagine only and hybrid tasks datasets respectively as illustrated in Figure 7, Figure 8, and Figure 9.

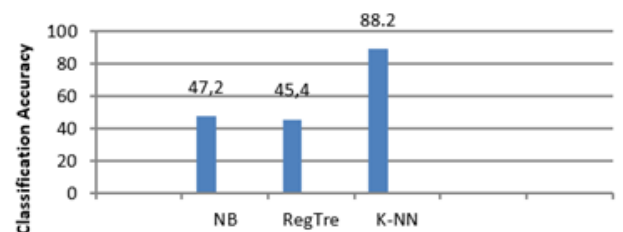

Figure 7. Classification results for BCI competition IV-a

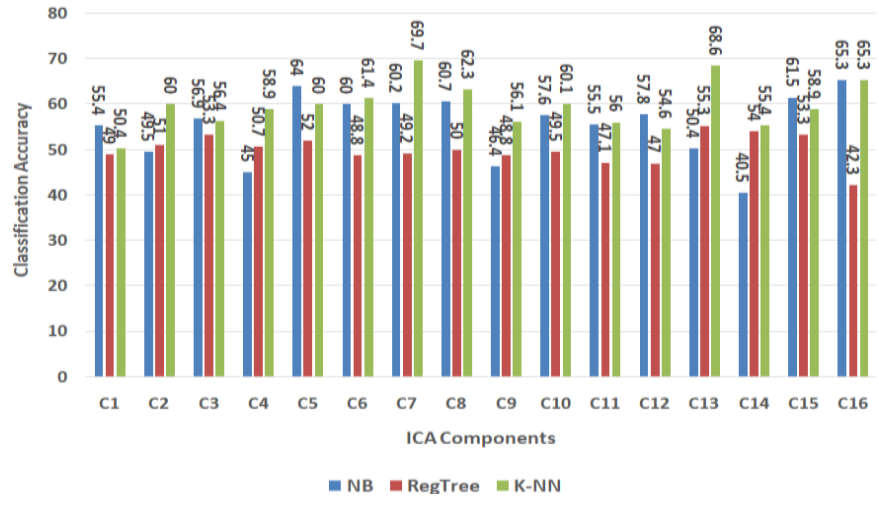

Figure 8. Classification results for imagine only EEG dataset

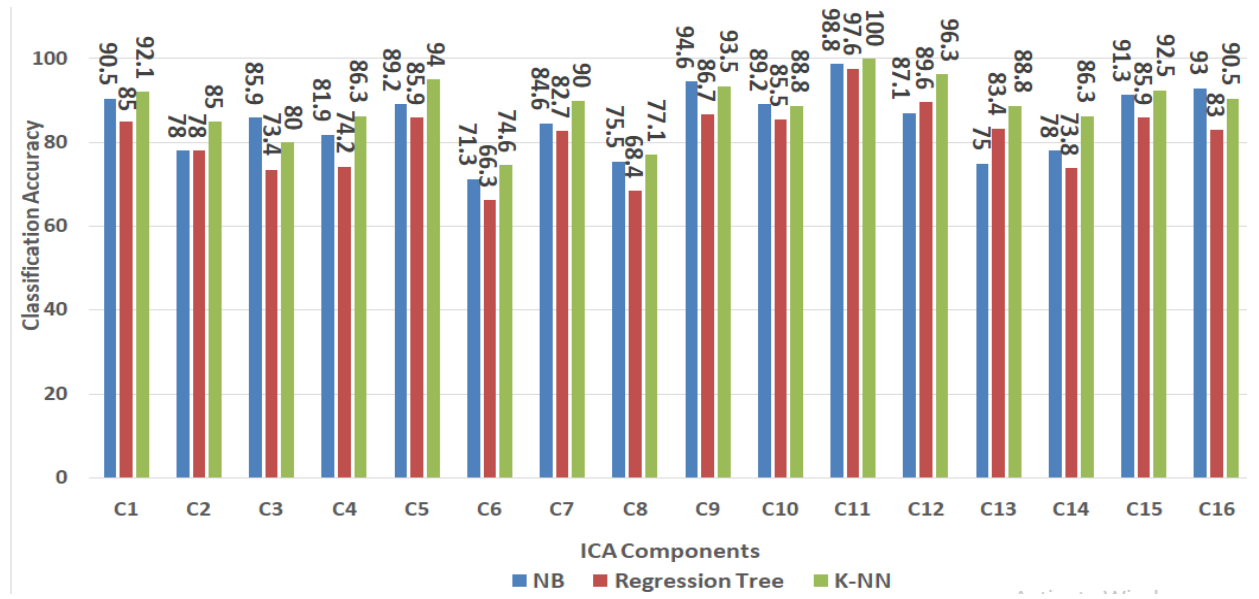

Figure 9. Classification results for hybrid tasks EEG dataset

\subsection{Robotic ARM control}

In this section, the success rate of each of both MI tasks and hybrid tasks was evaluated. Consequently, hybrid task obtained the highest success rate for each class as illustrated in Table 4. As such hybrid tasks were utilized to validate predicted classes through dobot magician robotic arm control. MATLAB was interfaced with arduino board via a serial communication port, whereby pins (Tx, Rx, GND and $5 \mathrm{~V}$ ) on the arduino board were connected to pins (Rx, Tx, GND and 5V) on the communication interface of the robotic arm respectively. MATLAB script was utilized to send any of the predicted classes to Arduino board. The PTP mode was set to MOVL on arduino script. Whenever arduino script received a character value (class label) from MATLAB script, four robotic movement (left, right, up and down) corresponding to each predicted class were executed.

Factors influencing low intension detection rate in a non-invasive EEG-based ... (Clifford Maswanganyi) 
Table 4. Success rate for each MI and hybrid task

\begin{tabular}{c} 
Class Left Right Up Down Average \\
\hline MI Tasks (Competition) $80.92 \% 87.03 \% 94.66 \% 90.3 \% 88.2 \%$ \\
MI Tasks (Online recorded) $57.45 \% 55.85 \% 72.5 \% 63.58 \% 62.3 \%$ \\
Hybrid Tasks (Online recorded) $97.22 \% 88.95 \% 94.74 \% 95.79 \% 94.17 \%$ \\
\hline
\end{tabular}

\section{CONCLUSION}

A hybrid task EEG-based BCI system was presented in this study with an objective of improving MI task detection rate by investigating the impact of artifacts, extracted feature dimension and visual stimulus on MI task detection rate. Three EEG datasets consisting of MI and hybrid tasks respectively, were utilized to facilitate the investigation. A runICA algorithm was then utilized to eliminate the impact of artifacts on all filtered EEG datasets. FFT and WPT algorithms were then applied on artifact free components to extract three sets of features, from which the DEFS algorithm eliminated the impact of dimensionality by selecting relevant feature subsets. Moreover, the selected feature subsets were utilized as input parameters to three classifiers (K-NN, NB and RegTree). Consequently, K-NN classifier obtained a highest intention detection rate of $100 \%$ for hybrid tasks. Moreover, accurately predicted hybrid tasks were validated through a robotic arm control. As such this study has conclusively proven that visual stimulus, artifacts free ICA components and DEFS selected high predictive feature subsets can significantly enhence MI task prediction rate in BCI system.

\section{REFERENCES}

[1] A. Mahmood, R. Zainab, R. B. Ahmad, M. Saeed, and A. M. Kamboh, "Classification of multi-class motor imagery EEG using four band common spatial pattern," in 2017 39th Annual International Conference of the IEEE Engineering in Medicine and Biology Society (EMBC), pp. 1034-1037, 2017.

[2] R. Roy, et al., "EEG based motor imagery study of time domain features for classification of power and precision hand grasps," in 2017 8th International IEEE/EMBS Conference on Neural Engineering (NER), pp. 440-443, 2017.

[3] M. E. Sharbaf, A. Fallah, and S. Rashidi, "EEG-based multi-class motor imagery classification using variable sized filter bank and enhanced One Versus One classifier," in 2017 2nd Conference on Swarm Intelligence and Evolutionary Computation (CSIEC), pp. 135-140, 2017.

[4] R. Chatterjee and T. Bandyopadhyay, "EEG based Motor Imagery Classification using SVM and MLP," in 2016 2nd International Conference on Computational Intelligence and Networks (CINE), pp. 84-89, 2016.

[5] L. F. Nicolas-Alonso, R. Corralejo, J. Gomez-Pilar, D. Álvarez, and R. Hornero, "Adaptive stacked generalization for multiclass motor imagery-based brain computer interfaces," IEEE Transactions on Neural Systems and Rehabilitation Engineering, vol. 23, no. 4, pp. 702-712, 2015.

[6] C. Maswanganyi, et al., "Discrimination of Motor Imagery Task using Wavelet Based EEG Signal Features," International Conference on Intelligent and Innovative Computing Applications (ICONIC), pp. 1-4, 2018.

[7] A. Myrden and T. Chau, "A passive EEG-BCI for single-trial detection of changes in mental state," IEEE Transactions on neural systems and rehabilitation Engineering, vol. 25, no. 4, pp. 345-356, 2017.

[8] X. Wang, T. Cao, B. Wang, F. Wan, P. U. Mak, P. I. Mak, et al., "An online SSVEP-based chatting system," in Proceedings 2011 International Conference on System Science and Engineering, pp. 536-539, 2011.

[9] B. A. Osuagwu, et al., "Is implicit motor imagery a reliable strategy for a brain-computer interface?," IEEE Transactions on Neural Systems and Rehabilitation Engineering, vol. 25, no. 12, pp. 2239-2248, 2017.

[10] H. Ghandeharion, et al., "A fully automatic ocular artifact suppression from EEG data using higher order statistics: improved performance by wavelet analysis," Medical engineering \& physics, vol. 32, no. 7, pp. 720-729, 2010.

[11] L. Bi, X.-A. Fan, and Y. Liu, "EEG-based brain-controlled mobile robots: A survey," IEEE transactions on humanmachine systems, vol. 43, no. 2, pp. 161-176, 2013.

[12] X. Xie, Z. L. Yu, H. Lu, Z. Gu and Y. Li, "Motor imagery classification based on bilinear sub-manifold learning of symmetric positive-definite matrices," in IEEE Transactions on Neural Systems and Rehabilitation Engineering, vol. 25, no. 6, pp. 504-516, 2017.

[13] T. Mladenov, K. Kim, and S. Nooshabadi, "Accurate motor imagery based dry electrode brain-computer interface system for consumer applications," IEEE 16th International Symposium on Consumer Electronics, pp. 1-4, 2012.

[14] E. F. González-Castañeda, et al., "Sonification and textification: Proposing methods for classifying unspoken words from EEG signals," Biomedical Signal Processing and Control, vol. 37, pp. 82-91, 2017.

[15] N. A. Abduallatif, et al., "Brain-Computer Interface controlled functional electrical stimulation system for paralyzed arm," in 2016 8th Cairo International Biomedical Engineering Conference (CIBEC), pp. 48-51, 2016.

[16] S. Mantri, D. Patil, P. Agrawal, and V. Wadhai, "Non invasive EEG signal processing framework for real time depression analysis," in 2015 SAI Intelligent Systems Conference (IntelliSys), pp. 518-521, 2015.

[17] S. O'Regan, S. Faul, and W. Marnane, "Automatic detection of EEG artefacts arising from head movements using EEG and gyroscope signals," Medical engineering \& physics, vol. 35, no. 7, pp. 867-74; discussion 867, 2013.

[18] A. Dey, S. Bhattacharjee, and D. Samanta, "Recognition of motor imagery left and right hand movement using EEG," in 2016 IEEE International Conference on Recent Trends in Electronics, Information \& Communication Technology (RTEICT), pp. 426-430, 2016. 
[19] M. S. A. Arani and S. Moharreri, "Detection of imagination of fist movement using Analysis of occurrence sequence of EEG points in Poincare plot," in 2016 International Conference on Bio-engineering for Smart Technologies (BioSMART), pp. 1-4, 2016.

[20] J. Kevric and A. Subasi, "Comparison of signal decomposition methods in classification of EEG signals for motorimagery BCI system," Biomedical Signal Processing and Control, vol. 31, pp. 398-406, 2017.

[21] Y. U. Khan and F. Sepulveda, "Brain-computer interface for single-trial EEG classification for wrist movement imagery using spatial filtering in the gamma band," IET Signal Processing, vol. 4, no. 5, pp. 510-517, 2010.

[22] H. Zhang, H. Yang, and C. Guan, "Bayesian learning for spatial filtering in an EEG-based brain-computer interface," IEEE transactions on neural networks and learning systems, vol. 24, no. 7, pp. 1049-1060, 2013.

[23] S. Z. Bong, K. Wan, M. Murugappan, N. M. Ibrahim, Y. Rajamanickam, and K. Mohamad, "Implementation of wavelet packet transform and non linear analysis for emotion classification in stroke patient using brain signals," Biomedical Signal Processing and Control, vol. 36, pp. 102-112, 2017.

[24] E. Alickovic, J. Kevric, and A. Subasi, "Performance evaluation of empirical mode decomposition, discrete wavelet transform, and wavelet packed decomposition for automated epileptic seizure detection and prediction," Biomedical Signal Processing and Control, vol. 39, pp. 94-102, 2018.

[25] R. N. Khushaba, A. Al-Jumaily, and A. Al-Ani, "Novel feature extraction method based on fuzzy entropy and wavelet packet transform for myoelectric control," in 2007 International Symposium on Communications and Information Technologies, pp. 352-357, 2007.

[26] R. N. Khushaba, A. Al-Ani, and A. Al-Jumaily, "Feature subset selection using differential evolution and a statistical repair mechanism," Expert Systems with Applications, vol. 38, no. 9, pp. 11515-11526, 2011.

[27] L. Qin, B. Kamousi, Z. Liu, L. Ding, and B. He, "Classification of motor imagery tasks by means of timefrequency-spatial analysis for brain-computer interface applications," in Conference Proceedings. 2nd International IEEE EMBS Conference on Neural Engineering, pp. 374-376, 2005.

[28] R. Masoomi and A. Khadem, "Enhancing LDA-based discrimination of left and right hand motor imagery: Outperforming the winner of BCI Competition II," in 2015 2nd International Conference on Knowledge-Based Engineering and Innovation (KBEI), pp. 392-398, 2015.

[29] J. Kronegg, G. Chanel, S. Voloshynovskiy, and T. Pun, "EEG-based synchronized brain-computer interfaces: A model for optimizing the number of mental tasks," IEEE Transactions on Neural Systems and Rehabilitation Engineering, vol. 15, no. 1, pp. 50-58, 2007.

[30] X. Guo, et al., "Envelope detection based on online ICA algorithm and its application to motor imagery classification," in 2013 6th International IEEE/EMBS Conference on Neural Engineering, pp. 1058-1061, 2013.

\section{BIOGRAPHIES OF AUTHORS}
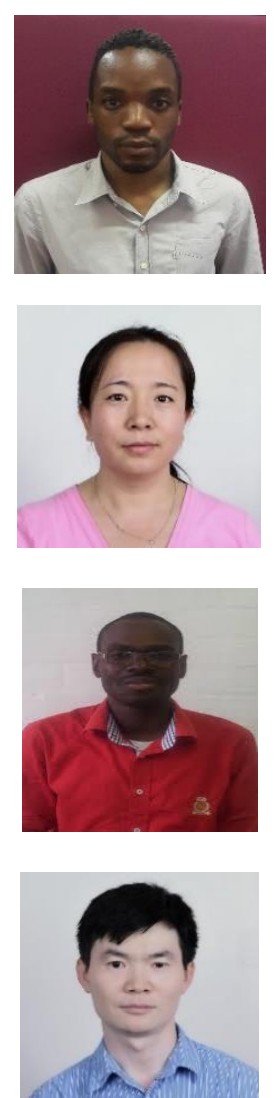

Clifford Rito Maswanganyi obtained Diploma of Computer Systems Engineering in 2014 and Baccalaureus Technologiae of Computer Systems Engineering in 2016 from Tshwane University of Technology. He finished his study in MCOM. In Computer Systems Engineering from Tshwane University of Technology in 2020. Now, he is currently pursuing his Phd in Computer Systems Engineering in the Faculty of Information and Communication Technology, Tshwane University of Technology, South Africa. His research interest is in signal processing, pattern recognition and brain computer interface.

Dr Chunling Tu received the Bachelor degree of computer science from Tianjin University of Technology and Education, China in 2002; MTech and MSc degrees in Electrical Engineering from Tshwane University of Technology (South Africa) and ESIEE Paris University (France) in 2010; DTech and PhD degrees of Electrical Engineering from Tshwane university of Technology and University Paris East, France in 2015. She is currently a senior lecturer at Tshwane University of Technology. Her research interests include image processing, AI, industrial control, machine learning, deep learning and pattern recognition.

Pius A. Owolawi (PhD) received his undergraduate degree in 2001 from the Federal University of Technology, Akure, Nigeria and also bagged his Master's and PhD Electrical Engineering from University of Kwazulu Natal, South Africa in 2006 and 2010 respectively. He is currently the Head of Department of Computer Systems Engineering, Tshwane University of Technology, South Africa. His research interests include, RF, Green communication, radiowave propagation (Microwave/ Millimeter wave systems), Satellite and free space optical communications, IOT, Embedded systems, Machine learning and data analytics.

Shengzhi Du received the M.S. degree in control theory and control engineering from Tianjin Poly Technology University, Tianjin, China, in 2001 and the Ph.D. degree in control theory and control engineering from Nankai University, Tianjin, China, in 2005. He is currently a professor in French South Africa Institute of Technology (F'SATI), Tshwane University of Technology, South Africa. His research interests include computer vision, AI, pattern recognition, and Human in the loop systems. 